\title{
Fourier Method for Approximating Eigenvalues of Indefinite Stekloff Operator ${ }^{\star}$
}

\author{
Yangqingxiang Wu and Ludmil Zikatanov $\bowtie$ \\ Department of Mathematics, The Pennsylvania State University \\ University Park, PA 16802, USA \\ ludmil@psu.edu \\ http://personal.psu.edu/ltz1/
}

\begin{abstract}
We introduce an efficient method for computing the Stekloff eigenvalues associated with the Helmholtz equation. In general, this eigenvalue problem requires solving the Helmholtz equation with Dirichlet and/or Neumann boundary condition repeatedly. We propose solving the related constant coefficient Helmholtz equation with Fast Fourier Transform (FFT) based on carefully designed extensions and restrictions of the equation. The proposed Fourier method, combined with proper eigensolver, results in an efficient and clear approach for computing the Stekloff eigenvalues.
\end{abstract}

Keywords: Stekloff Eigenvalues, FFT, Helmholtz Equation

\section{Introduction}

We consider the problem of computing the Stekloff eigenvalues corresponding to the indefinite Helmholtz equation. The efficient computation of such eigenvalues is needed in several numerical models. For example, in inverse scattering, as discussed in Cakoni et al. 2016, these eigenvalues carry information of the refractive index of an obstacle. We introduce the following boundary value problem: For a fixed $\eta$, find $\lambda \in \mathbb{C}$ such that there is a non-trivial solution $w \in H^{1}(\Omega)$ of the equation

$$
\mathcal{L}(\alpha, \lambda ; \eta):=\left\{\begin{array}{l}
-\Delta w-\eta^{2} w=0 \quad \text { in } \quad \Omega \\
\alpha \frac{\partial w}{\partial n}+\lambda w=0 \text { on } \quad \Gamma=\partial \Omega .
\end{array}\right.
$$

We call $\lambda$ a Stekloff eigenvalue when $\alpha=1$ and (1) has non-trivial solution.

As pointed out in Cakoni et al. [2016], the efficient computation of Stekloff eigenvalues is a challenging task. In addition, another important application of the techniques that we propose here is of interest in computing transmission eigenvalues where the aim is to find the kernel of the difference of two indefinite

\footnotetext{
* This work was supported in part by National Science Foundation Grants DMS1522615 and DMS-1720114
} 
Stekloff operators Cakoni and Kress 2017. The efficient solution of such problems, whether direct or inverse, requires fast solution of the Dirichlet problem, corresponding to the operator $\mathcal{L}(0,1 ; k(x))$, and the Neumann problem, corresponding to the operator $\mathcal{L}(1,0 ; k(x))$ in (1). Here, $k(x)$ is the wave number and in case of non homogenous problem, such as $\mathcal{L}(\cdot, \cdot ; \cdot)=(f, 0)^{t}$, the data $f(x)$ is the external force.

The difficulties associated with solving the indefinite Helmholtz equation numerically, especially in high frequency regimes, are well known (see Brandt and Livshits 1997, Ernst and Gander 2012]). Traditional iterative methods, such as Krylov subspace methods or standard MultiGrid (MG) and Domain Decomposition (DD) methods, are inefficient. We refer to Brandt and Livshits 1997, Ernst and Gander 2012 for discussion of such issues and guidance on how to tackle them.

Over the last two decades, different preconditioners and solvers for the Helmholtz equation have been proposed. We refer to the classical works by Brandt and Livshits Brandt and Livshits 1997], and Elman et al. Elman et al. [2001] for MG solvers and also to the more recent developments in Helmholtz preconditioning presented in Gander et al. [2015], Osei-Kuffuor and Saad 2010. More recent, Enquist and Ying Engquist and Ying $\mid 2011$ introduced the so called sweeping preconditioners which were further extended by Eslaminia and Guddati Eslaminia and Guddati 2016] to double-sweeping preconditioners. Stolk Stolk 2013 proposed a DD preconditioner based on special transmission conditions between subdomains. Other DD methods are found in Chen and Xiang 2013, ZepedaNúnez and Demanet 2016].

In our focus are the computations of Stekloff eigenvalues and the techniques which we propose here lead to efficient algorithms in many cases of practical interest and provide preconditioners for the Helmholtz problem. More importantly, our techniques easily extend to the Maxwell's system because they are based on the Fourier method.

The rest of the paper is organized as follows. We introduce the Fourier method for solving the constant coefficients boundary value problem in Section 2.1 (Dirichlet) and in Section 2.2 (Neumann). Further, in Section 3, we formulate the Stekloff eigenvalue problem and show how the FFT based Helmholtz solver can be applied. We conclude with several numerical tests on Stekloff eigenvalue computations as well as solution of the Helmholtz equation with variable wave number.

\section{Periodic Extensions and Fourier Method}

\subsection{Dirichlet Boundary Conditions: 1d Case}

To explain the ideas we consider the $1 \mathrm{D}$ version of $(1)$ in the interval $(0,1)$ :

$$
-u^{\prime \prime}-k^{2} u=f, \quad u(0)=u(1)=0 .
$$


After a discretization, using central finite difference, we obtain the following linear system

$$
A^{D} \boldsymbol{u}=\boldsymbol{f}, \quad A^{D}=T^{D}-k^{2} h^{2} I \in \mathbb{R}^{n \times n},
$$

where $T^{D}=\operatorname{diag}(-1,2,-1)$ is a tri-diagonal matrix, $\boldsymbol{u}=\left(u_{1}, \ldots, u_{n}\right)^{t}, u_{0}=$ $u_{n+1}=0, h=1 /(n+1), \boldsymbol{f}=h^{2}\left(f_{1}, \ldots, f_{n}\right)^{t}$, and $u_{j} \approx u(j h), f_{j} \approx f(j h)$, $j=1, \ldots n$.

Let us now consider the same equation on a larger domain $(0,2)$ and with periodic boundary conditions:

$$
-v^{\prime \prime}-k^{2} v=g, \quad v(0)=v(2), \quad v^{\prime}(0)=v^{\prime}(2) .
$$

Analogous discretization approach leads to a linear system for $\boldsymbol{v}=\left(v_{1}, \ldots, v_{N}\right)^{t}$, $N=2 n+2$, which is as follows:

$$
A^{P} \boldsymbol{v}=\boldsymbol{g}, \quad A^{P}=T^{P}-k^{2} h^{2} I \in \mathbb{R}^{N \times N} .
$$

Here, $e_{1}=(1,0, \ldots, 0)^{t}$ and $e_{N}=(0, \ldots, 0,1)^{t}$ are the standard Euclidean basis vectors and $T^{P}=\operatorname{diag}(-1,2,-1)-e_{1} e_{N}^{t}-e_{N} e_{1}^{t}$ is a circulant matrix. The right hand side $\boldsymbol{g}=h^{2}\left(g_{1}, \ldots, g_{N}\right)^{t}$ is a given vector in $\mathbb{R}^{N}$ depending on $\boldsymbol{f}$, which we specify later. The unknowns in this case are $v_{j} \approx v(2 j / N), j=1, \ldots, N$. Notice that from the periodic boundary conditions, we have $v_{N} \approx v(0)=v(2)$ and $v_{1} \approx v(2 / N)$ and this is reflected in the first and the last equation in the linear system (4).

The solution of systems with circulant matrices can be done efficiently using the Fast version (FFT) of the Discrete Fourier Transform (DFT) (see Cooley and Tukey 1965 for a description of FFT). The DFT is represented by an operator $\mathcal{F}: \mathbb{C}^{N} \rightarrow \mathbb{C}^{N}$ represented by a matrix (denoted again with $\mathcal{F}$ ) defined as: $\mathcal{F}_{j m}=\omega^{(j-1)(m-1)}, \quad \omega=e^{-\frac{2 i \pi}{N}}$, andj $=1, \ldots, N, \quad m=1, \ldots, N$. As is well known, we have the DFT inversion formula:

$$
\mathcal{F}^{-1}=\frac{1}{N} \mathcal{F}^{*}=\frac{1}{N} \overline{\mathcal{F}}
$$

Since $A^{P}$ here is a circulant matrix it is diagonalized by $\mathcal{F}$ Cooley and Tukey 1965, Golub and Van Loan 2012 and

$$
\mathcal{F} A^{P} \mathcal{F}^{-1}=D^{P}=\operatorname{diag}\left(d_{l}\right), \quad d_{l}=4 \sin ^{2} \frac{\pi(l-1)}{N}-k^{2} h^{2}, \quad l=1, \ldots N .
$$

As a consequence of this proposition, the solution $\boldsymbol{v}$ to the problem (4) can be obtained by

$$
\boldsymbol{v}=\mathcal{F}^{-1}\left(D^{P}\right)^{-1} \mathcal{F} \boldsymbol{g} .
$$

Let us now consider the special case when $\boldsymbol{g}$ in (4) corresponds to an "odd" function. We have the following simple result.

Proposition 1. If $N=2 n+2$ and $\boldsymbol{g}$ satisfies $g_{j}=-g_{N-j}$, for $j=n+2, n+$ $3, \ldots, 2 n+1$ and $g_{n+1}=g_{2 n+2}=0$, then the solution to (4) satisfies the relation:

$$
v_{j}=-v_{N-j}, \quad j=n+2, \ldots, 2 n+1 .
$$


Proof. We note that by assumption, $\boldsymbol{g}$ is an "odd" function with respect to the middle of the interval $(0,2)$. Since $A^{D}$ is an invertible matrix, let $\boldsymbol{u}$ satisfy $\left[A^{D} \boldsymbol{u}\right]_{j}=\boldsymbol{g}_{j}, j=1, \ldots, n$. Next, we define $\boldsymbol{v}=E \boldsymbol{u} \in \mathbb{C}^{N}$ where $E$ is the extension operator defined below in (8) and it is immediate to verify that $A^{P} \boldsymbol{v}=\boldsymbol{g}$. Since $A^{P}$ is also invertible, $\boldsymbol{v}$, which satisfies this relation, is the unique solution of $A^{P} \boldsymbol{v}=\boldsymbol{g}$. From the definition of $E$, we conclude that $\boldsymbol{v}$ also satisfies (7).

Based on this observation, to solve the Dirichlet problem, we can define a linear operator $B$ (which we will soon prove equals $\left(A^{D}\right)^{-1}$ ) as follows: Let the extension $\boldsymbol{g}:=E \boldsymbol{f}$ be defined as

$$
\mathbb{C}^{N} \ni \boldsymbol{g}=E \boldsymbol{f}, \quad g_{j}=\left\{\begin{array}{l}
f_{j}, \quad j=1, \ldots, n, \\
0, \quad j=n+1, \\
-f_{N-j}, \quad j=n+2, \ldots, 2 n+1 \\
0, \quad j=N .
\end{array}\right.
$$

Here, $N=2 n+2$ and we also have the following restriction operator

$$
\mathbb{C}^{n} \ni \boldsymbol{w}=R \boldsymbol{v}, \quad w_{j}=v_{j+1}, \quad j=1, \ldots n, \quad \boldsymbol{v} \in \mathbb{R}^{N} .
$$

We then set

$$
B \boldsymbol{f}=R \mathcal{F}^{-1}\left(D^{P}\right)^{-1} \mathcal{F} E \boldsymbol{f} .
$$

As the next proposition shows, $B$ provides the exact solution to problem (2).

Proposition 2. With $B$ defined in 10 we have

$$
B=\left(A^{D}\right)^{-1}, \quad \text { or equivalently, } \quad \boldsymbol{u}=B \boldsymbol{f} .
$$

Proof. We notice that $R=\left(I_{n} \mid \mathbf{0}\right) \in \mathbb{R}^{n \times N}$, and $E=\left(I_{n}, \mathbf{0}_{n \times 1},-\tilde{I}_{n}, \mathbf{0}_{n \times 1}\right)^{T} \in$ $\mathbb{R}^{N \times n}$, where $I_{n}$ is the $n \times n$ identity matrix and $\tilde{I}_{n}=\left(\delta_{i, n+1-j}\right)_{i j}$. Computing the product $A^{D} R\left(A^{P}\right)^{-1} E$ then shows that:

$$
A^{D} R\left(A^{P}\right)^{-1} E=A^{D}(I \mid 0)\left(A^{P}\right)^{-1} E=(A \mid 0)\left(A^{P}\right)^{-1} E=(I \mid 0) E=I .
$$

Indeed, the identities in (11) are verified by direct calculation:

$$
\begin{aligned}
& \left((A \mid 0) A_{p}^{-1}\right)_{i j} \\
& =-\frac{1}{N}\left(\mathcal{F}^{-1} D_{p}^{-1} \mathcal{F}\right)_{i-1, j}+\frac{2}{N}\left(\mathcal{F}^{-1} D_{p}^{-1} \mathcal{F}\right)_{i, j}-\frac{1}{N}\left(\mathcal{F}^{-1} D_{p}^{-1} \mathcal{F}\right)_{i+1, j} \\
& =\frac{1}{N} \sum_{l=1}^{N}\left(-\bar{\omega}^{(i-2)(l-1)}+\left(2-k^{2} h^{2}\right) \bar{\omega}^{(i-1)(l-1)}-\bar{\omega}^{i(l-1)}\right) d_{l} \omega^{(k-1)(j-1)} \\
& =\frac{1}{N} \sum_{l=1}^{N}\left(2-k^{2} h^{2}-2 \cos \left(\frac{2 \pi(l-1)}{N}\right)\right) d_{l} \omega^{(k-1)(j-i)} \\
& =\frac{1}{N} \sum_{l=1}^{N} \omega^{(k-1)(j-i)}=\delta_{i j} .
\end{aligned}
$$

This completes the proof. 
Generalization to Higher Dimensions (Dirichlet Problem) We now consider the Helmholtz operator $\mathcal{L}(0,1 ; k(x))$, defined in (1) in $d$-dimensions, i.e. we take $\Omega=(0,1)^{d}$. Discretization with standard central finite differences, we have the linear system:

$$
A^{D} \boldsymbol{u}=\boldsymbol{f}, \quad A^{D}=\sum_{j=1}^{d}\left(I^{\otimes(j-1)} \otimes T^{D} \otimes I^{\otimes(d-j)}\right)-k^{2} h^{2} I^{\otimes d} \in \mathbb{R}^{n^{d} \times n^{d}} .
$$

Here $M^{\otimes p}:=\underbrace{M \otimes \ldots \otimes M}_{p \text { copies }}$ for any matrix $M$ and $T^{D}$ as in $(2)$ is the triangular matrix.

The extension and restriction operators in higher dimensions can be written as $E_{d}:=E^{\otimes d}$ and $R_{d}:=R^{\otimes d} \cdot \boldsymbol{g}=E_{d} \boldsymbol{f}$ is the "odd" extension of $\boldsymbol{f}$. As in the $1 \mathrm{D}$ case, the linear system for the extended Helmholtz equation is:

$$
A^{P} \boldsymbol{v}=\boldsymbol{g}, \quad A^{P}=\sum_{j=1}^{d}\left(I^{\otimes(j-1)} \otimes T^{P} \otimes I^{\otimes(d-j)}\right)-k^{2} h^{2} I^{\otimes d} \in \mathbb{R}^{N^{d} \times N^{d}},
$$

where $T^{P}$ has been defined in (4). As in the one dimensional case (5), the matrix $A^{P}$ is diagonalized by the multidimensional DFT $\mathcal{F}_{d}=\mathcal{F}^{\otimes d}$. The multidimensional version of (5) then is:

$$
\mathcal{F}_{d} A^{P} \mathcal{F}_{d}^{-1}=D_{d}^{P}:=\sum_{j=1}^{d}\left(I^{\otimes(j-1)} \otimes D^{P} \otimes I^{\otimes(d-j)}\right)-k^{2} h^{2} I^{\otimes d},
$$

As a consequence, we obtain inversion formula similar to the one presented in Proposition 2. To show such representation, we need the following result.

Lemma 1. Let $E$ and $R$ be extension and restriction operator defined in (8) and $(9)$ respectively. Then following identity holds

$$
A^{D}=R^{\otimes d} A^{P} E^{\otimes d} .
$$

Proof. By using the standard properties of the tensor product, we have

$$
\begin{aligned}
R^{\otimes d} A^{P} E^{\otimes d} & =R^{\otimes d}\left(\sum_{j=1}^{d}\left(I^{\otimes(j-1)} \otimes T^{P} \otimes I^{\otimes(d-j)}\right)-k^{2} h^{2} I^{\otimes d}\right) E^{\otimes d} \\
& =R^{\otimes d}\left(\sum_{j=1}^{d}\left(E^{\otimes(j-1)} \otimes T^{P} E \otimes E^{\otimes(d-j)}\right)-k^{2} h^{2} E^{\otimes d}\right) \\
& =\sum_{j=1}^{d}\left(I^{\otimes(j-1)} \otimes R T^{P} E \otimes I_{n-1}^{\otimes(d-j)}\right)-k^{2} h^{2} I^{\otimes d} .
\end{aligned}
$$

It is straightforward to check that $R T^{P} E=T^{D}$. Thus, $R^{\otimes d} A^{P} E^{\otimes d}=A^{D}$. 
The following theorem gives the representation of the inverse of the discretized Dirichlet problem in the multidimensional case.

Theorem 1. The inverse of $A^{D}$ can be written as

$$
\left(A^{D}\right)^{-1}=R_{d}\left(A^{P}\right)^{-1} E_{d}, \quad \text { or equivalently, } \quad \boldsymbol{u}=R_{d}\left(A^{P}\right)^{-1} E_{d} \boldsymbol{f} .
$$

Proof. Clearly, it is sufficient to prove that $A^{D} R_{d}\left(A^{P}\right)^{-1} E_{d}=I$.

$$
A^{D} R_{d}\left(A^{P}\right)^{-1} E_{d}=R_{d} A^{P} E_{d} R_{d}\left(A^{P}\right)^{-1} E_{d}=R^{\otimes d} A^{P} E^{\otimes d} R^{\otimes d}\left(A^{P}\right)^{-1} E^{\otimes d}=I,
$$

by the using properties of the matrix tensor product, Lemma 1 and identity $R E=I$.

Notice here, all the above results can be extended to rectangle (non-square) domain without too much difference. As a conclusion, we can solve the constant coefficient Helmholtz equation with Dirichlet boundary condition by FFT with complexity $O(n \lg n)$, where $n$ is the problem size.

\subsection{Neumann Boundary Conditions}

We next discuss the Fourier method for solving the Neumann problem. We begin by laying out the details for $1 \mathrm{D}$ case.

Neumann Problem in 1D Let's consider the following 1D Helmholtz equation with one side Neumann boundary condition in $(0,1)$ :

$$
-u^{\prime \prime}-k^{2} u=f, \quad u(0)=0, \quad u^{\prime}(0)=g .
$$

Finite difference discretization gives the linear system of equations $A \boldsymbol{u}=\boldsymbol{F}$ where

$$
A=\operatorname{diag}\left(-1,2-k^{2} h^{2},-1\right)-\left(1-\frac{1}{2} k^{2} h^{2}\right) e_{n+1} e_{n+1}^{t} \in \mathbb{R}^{(n+1) \times(n+1)},
$$

and also $\boldsymbol{u}=\left(u_{1}, \ldots, u_{n+1}\right)^{t}, h=1 /(n+1), u_{j} \approx u(j h), f_{j} \approx f(j h)$, for $j=1, \ldots n+1$, and $\boldsymbol{F}=\left(h^{2} f_{1}, \ldots, h^{2} f_{n}, \frac{1}{2} h^{2} f_{n+1}+h g\right)^{t}$.

With Fourier method for the Dirichlet problem in mind, we do "even" extension of the system (18) to get a Toeplitz system similar to (2):

$$
A^{e} \boldsymbol{u}^{e}=\boldsymbol{F}^{e}, \quad A^{e}=\operatorname{diag}\left(-1,2-k^{2} h^{2},-1\right) \in \mathbb{R}^{M \times M}
$$

where $M=2 n+1, \boldsymbol{u}^{e}=\left(u_{1}, \ldots, u_{M}\right)^{t}$, and $\boldsymbol{F}^{e}=\left(h^{2} f_{1}, \ldots, h^{2} f_{n}, h^{2} f_{n+1}+\right.$ $\left.2 h g, h^{2} f_{n}, \ldots, h^{2} f_{1}\right)^{t}$. By symmetry, the solution of system $(19)$, when restricted on interval $(0,1)$, will be the same as solution of system (18). Even though the problem size has been doubled, the extended system is Toeplitz, thus can be solved by Fourier method from Section 2.1. 
In summary, we have the following inverse of the Neumann operator, that is the solution of :

$$
A^{-1} \boldsymbol{F}=R \mathcal{F}^{-1}\left(D^{p}\right)^{-1} \mathcal{F} E_{o} E_{e} \boldsymbol{F}
$$

The operators involved in the definition above are (from right to left): even extension, odd extension followed by the inverse of the periodic problem and then restriction. More precisely, for the even extension $E_{e}$ we have,

$$
\mathbb{C}^{M} \ni \quad\left[E_{e} \boldsymbol{F}\right]_{j}=\left\{\begin{array}{l}
F_{j}, \quad j=1, \ldots, n, \\
2 F_{n+1}, \quad j=n+1, \\
F_{M+1-j}, \quad j=n+2, \ldots, 2 n+1 .
\end{array}\right.
$$

Next, for the extension as to odd functions/vectors we have:

$$
\mathbb{C}^{P} \ni\left[E_{o} E_{e} \boldsymbol{F}\right]_{j}=\left\{\begin{array}{l}
F_{j}^{e}, \quad j=1, \ldots, M, \\
0, \quad j=M+1, \\
-F_{N-j}^{e}, \quad j=M+2, \ldots, 2 M+1 \\
0, \quad j=2 M+2 .
\end{array}\right.
$$

Finally, we have the diagonal $D^{p}=\operatorname{diag}(4 \sin (j \pi / M))_{j=0}^{M-1}$ and the restriction $R$ :

$$
\boldsymbol{v} \in \mathbb{R}^{P}, \quad \mathbb{C}^{n+1} \ni \boldsymbol{u}=R \boldsymbol{v}, \quad u_{j}=v_{j}^{p}, \quad j=1, \ldots n+1 .
$$

Generalization to Higher Dimensions (Neumann problem) The Fourier method for Neumann problem can also be generalized to any dimension $d$ in a fashion similar to the procedure given earlier for the Dirichlet problem. Just for illustration, if we consider $d=2$ and $\Omega=(0,1)^{2}$ with the following boundary conditions:

$$
\frac{\partial u}{\partial x}=g, \quad \text { on } \quad \Gamma_{1}=\{x=1\} \times(0,1)
$$

and homogeneous Dirichlet conditions elsewhere. To solve the resulting linear system we first do an "even" extension of the data and we arrive at:

$$
A^{e} \boldsymbol{u}^{e}=\boldsymbol{F}^{e}, \quad A^{e}=I_{M} \otimes T_{n}^{d}+T_{M}^{d} \otimes I_{n}-k^{2} h^{2} I_{M n} \in \mathbb{R}^{M n \times M n},
$$

where $T_{j}^{d}=\operatorname{tridiag}(-1,2,-1) \in \mathbb{R}^{j \times j}, M=2 n+1, \boldsymbol{u}^{e}=\left(\boldsymbol{u}_{1}, \ldots, \boldsymbol{u}_{M}\right)^{t}$, and $\boldsymbol{F}^{e}=\left(h^{2} \boldsymbol{f}_{1}, \ldots, h^{2} \boldsymbol{f}_{n}, h^{2} \boldsymbol{f}_{n+1}+2 h g, h^{2} \boldsymbol{f}_{n}, \ldots, h^{2} \boldsymbol{f}_{1}\right)^{t}$. Clearly, on $\Omega$, the restriction of $\boldsymbol{u}^{e}$ is the same as the solution $\boldsymbol{u}$ of the Neumann problem. Now, for the solution of 23 we can apply the method we have already described in 2.1 .

\section{Stekloff Eigenvalue Computation with Fourier Method}

\subsection{Variational Formulation}

Multiplying the first equation in (1) by $v \in H^{1}(\Omega)$ and integrating by parts, we get:

$$
\int_{\Omega} \nabla w \nabla v-\eta^{2} \int_{\Omega} w v=-\lambda \int_{\Gamma} w v
$$


Define $A(\eta): H^{1}(\Omega) \rightarrow H^{-1}(\Omega)$ as

$$
\langle A(\eta) w, v\rangle:=(\nabla w, \nabla v)_{\Omega}-\eta^{2}(w, v)_{\Omega} \quad \forall v \in H^{1}(\Omega),
$$

where $(\cdot, \cdot)_{\Omega}$ is the $L^{2}$ inner product and $\langle\cdot, \cdot\rangle$ is duality pairing between $H^{-1}(\Omega)$ and $H^{1}(\Omega)$. The Stekloff operator, or Dirichlet-to-Neumann(DtN), $S(\eta)$ can be defined in two steps:

Firstly, for any $f \in H^{1 / 2}(\Gamma)$, define $f_{0} \in H_{0}^{1}(\Omega)$ as the unique function satisfies:

$$
\left\langle A(\eta) f_{0}, v_{0}\right\rangle=-\left\langle A(\eta)(E f), v_{0}\right\rangle, \quad \forall v_{0} \in H_{0}^{1}(\Omega),
$$

where $E f$ is $H^{1}$-bounded extension of $f$, e.g. harmonic extension.

Secondly, define the action of $S(\eta): H^{1 / 2}(\Gamma) \rightarrow H^{-1 / 2}(\Gamma)$ as

$$
\langle S(\eta) f, g\rangle_{1 / 2}=\left\langle A(\eta)\left(f_{0}+E f\right), E g\right\rangle, \quad \forall g \in H^{1 / 2}(\Gamma),
$$

where $\langle\cdot, \cdot\rangle_{1 / 2}$ is the duality pairing between $H^{1 / 2}(\Gamma)$ and $H^{-1 / 2}(\Gamma)$.

Lemma 2. The equation (1) has a non-trivial solution $\Leftrightarrow \lambda$ is an eigenvalue of the Stekloff operator $S(\eta)$.

Proof. We prove one side of this equivalence here since the other half is similar. Suppose $u$ is solution to (1), then

$$
\left\langle A(\eta) u, v_{0}\right\rangle=0, \quad \forall v_{0} \in H_{0}^{1}(\Omega) .
$$

From the above equation, we have

$$
\left\langle A(\eta)\left(u-E u_{\Gamma}\right), v_{0}\right\rangle=-\left\langle A(\eta) E u_{\Gamma}, v_{0}\right\rangle \quad \forall v_{0} \in H_{0}^{1}(\Omega),
$$

where $u_{\Gamma}:=\left.u\right|_{\Gamma}$ as the trace of $u$ on $\Gamma$. Denote $u_{0}:=u-E u_{\Gamma}$. It's easy to see that $u_{0} \in H_{0}^{1}(\Omega)$ and the following equation holds:

$$
\left\langle S(\eta) u_{\Gamma}, g\right\rangle_{1 / 2}=\left\langle A(\eta)\left(u_{0}+E u_{\Gamma}\right), E g\right\rangle=\left(\lambda u_{\Gamma}, g\right) .
$$

This shows that $\lambda$ must be the eigenvalue of $S(\eta)$.

This lemma implies that solving problem (1) is equivalent to solving the eigenvalue problem for $S(\eta)$, for given $\eta$. In the next section we describe an efficient method for this task, namely the Fourier method for Stekloff eigenvalues.

\subsection{Neumann-to-Dirichlet and Dirichlet-to-Neumann Operators}

Let $\mu \in L^{2}(\Gamma)$ and define $w_{\mu}$ to be the solution of equation (1) with Neumann boundary condition, i.e. $w_{\mu}$ satisfies

$$
\left\langle A(\eta) w_{\mu}, v\right\rangle=\left\langle\mu, v_{\Gamma}\right\rangle_{1 / 2}, \quad \text { for any } \quad v \in H^{1}(\Omega) .
$$


Taking the trace of $w_{\mu}$, we can define Neumann-to-Dirichlet (NtD) operator $T$ as $T \mu=\left.w_{\mu}\right|_{\Gamma}$. After discretization, we have the following linear system:

$$
\left[\begin{array}{ll}
A_{I I} & A_{I B} \\
A_{B I} & A_{B B}
\end{array}\right]\left[\begin{array}{l}
\boldsymbol{w}_{I} \\
\boldsymbol{w}_{B}
\end{array}\right]=\left[\begin{array}{l}
\mathbf{0} \\
\boldsymbol{\mu}
\end{array}\right],
$$

where $\boldsymbol{w}_{I}$ and $\boldsymbol{w}_{B}$ are solution of 26 restricted in $\Omega$ and on $\Gamma$ respectively. Then the discretized NtD operator $T_{h}$ corresponding to the NtD operator $T$ is

$$
T_{h} \boldsymbol{\mu}=[\mathbf{0}, I]\left[\begin{array}{ll}
A_{I I} & A_{I B} \\
A_{B I} & A_{B B}
\end{array}\right]^{-1}\left[\begin{array}{l}
\mathbf{0} \\
\boldsymbol{\mu}
\end{array}\right] .
$$

To discretize the Stekloff operator $S$ in analogous fashion, we also consider the Dirichlet boundary problem of (1) with boundary data $f \in H^{1 / 2}(\Omega)$. With the same discretization and the same ordering as for the Neumann problem, we have the following linear system:

$$
\left[\begin{array}{cc}
A_{I I} & A_{I B} \\
\mathbf{0} & I
\end{array}\right]\left[\begin{array}{l}
\boldsymbol{w}_{I} \\
\boldsymbol{w}_{B}
\end{array}\right]=\left[\begin{array}{l}
\mathbf{0} \\
\boldsymbol{f}
\end{array}\right] .
$$

Clearly, $\boldsymbol{w}_{b}=\boldsymbol{f}$ as expected and the discrete version of 25 gives the action of $S_{h}$ as

$$
S_{h} \boldsymbol{f}=\left(A_{B B}-A_{B I} A_{I I}^{-1} A_{I B}\right) \boldsymbol{f} .
$$

We have the following Lemma, which shows that $T_{h}$ is the inverse of $S_{h}$.

Lemma 3. For the operators $T_{h}$ and $S_{h}$ defined in (28) and (30), respectively, we have $S_{h} T_{h}=I$.

Proof. We just need to show $S_{h} T_{h} \boldsymbol{\mu}=\boldsymbol{\mu}$ for any $\boldsymbol{\mu}$. This can be easily proved by Block-LU factorization.

$$
\begin{aligned}
S_{h} T_{h} \boldsymbol{\mu} & =S_{h}[\mathbf{0}, \boldsymbol{I}]\left[\begin{array}{ll}
A_{I I} & A_{I B} \\
A_{B I} & A_{B B}
\end{array}\right]^{-1}\left[\begin{array}{l}
\mathbf{0} \\
\boldsymbol{\mu}
\end{array}\right] \\
& =S_{h}[\mathbf{0}, \boldsymbol{I}]\left(\left[\begin{array}{cr}
I & \mathbf{0} \\
A_{B I} A_{I I}^{-1} & I
\end{array}\right]\left[\begin{array}{cc}
A_{I I} & A_{I B} \\
\mathbf{0} & S_{h}
\end{array}\right]\right)^{-1}\left[\begin{array}{l}
\mathbf{0} \\
\boldsymbol{\mu}
\end{array}\right]=\boldsymbol{\mu} .
\end{aligned}
$$

The results from the previous two sections show that we can efficiently compute the Stekloff eigenvalues of small magnitude as well as large magnitude. For example, the eigenvalues of small magnitude of $S_{h}$ can be approximated by the reciprocal of the eigenvalues of $\mathrm{NtD}$ operator $T_{h}$. The action of $T_{h}$, which is needed repeatedly in such a procedure, can be efficiently computed by the Fourier method applied to the solution of the Helmholtz-Neumann problem as we have discussed earlier. For the eigenvalues of largest magnitude the same applies, except that we need the action of $S_{h}$, which requires fast solution of the corresponding Dirichlet problems, which we also described earlier. 


\section{Numerical Examples}

\subsection{Helmholtz Equation with Varying Wave Number}

Until now, we have shown that Fourier method can solve the constant coefficient Helmholtz equation with Dirichlet boundary condition exactly with nearly linear complexity. Using this exact solver as preconditioner, we can also solve certain type of varying coefficient problem, e.g. slowly varying coefficient.

In this numerical example, we consider a homogeneous Dirichlet problem in domain $\Omega=(0,1)^{2}$ with uniform external force $f=1$ and the following velocity fields:

1. $c\left(x_{1}, x_{2}\right)=\frac{4}{3}\left[1-0.5 \exp \left(-0.5\left(x_{1}-0.5\right)^{2}\right]\right.$,

2. $c\left(x_{1}, x_{2}\right)=\frac{4}{3}\left[1-0.5 \exp \left(-0.5\left(x_{1}-0.5\right)^{2}+\left(x_{2}-0.5\right)^{2}\right)\right]$.

We study how the preconditioner behaves when $\omega$ and $n$ vary in the same ratio. In this way, the percentages of positive and negative eigenvalues are fixed, which poses the most challenge for computation. We record the numbers of iterations for GMRES method to converge (error tolerance $10^{-6}$ ) and total times used for computing in Table 1 . For $w / 2 \pi=3.2$, the wave number fields $k_{i}(x, y)$ and resulting wave field $u_{i}(x, y)$ with $i=1,2$ have been shown in Figure 1 .

Table 1. Varying $\omega$ and $n$, number of GMRES iterations and time to converge

\begin{tabular}{|c|c|c|c|c|c|}
\hline$\frac{\omega}{2 \pi}$ & $n^{2}$ & $N_{1}$ & $T_{1}$ & $N_{2}$ & $T_{2}$ \\
\hline 0.8 & $50^{2}$ & 4 & 0.068 & 4 & 0.062 \\
\hline 1.6 & $100^{2}$ & 5 & 0.074 & 5 & 0.076 \\
\hline 3.2 & $200^{2}$ & 6 & 0.12 & 6 & 0.12 \\
\hline 6.4 & $400^{2}$ & 13 & 0.60 & 10 & 0.46 \\
\hline
\end{tabular}

\subsection{Computing Stekloff Eigenvalues}

In this numerical example, we apply our Fourier-based method, which has been introduced in Section 3 to the Stekloff eigenvalues problem. We create a function handle that solves the Neumann boundary problem with FFT Helmholtz solver proposed, then use MATLAB eigs function to calculate the eigenvalues. For different $\eta$, the smallest six eigenvalues in magnitude can be found in Table 2 .

\section{Conclusions}

In this paper, we proposed an efficient method for finding eigenvalues of indefinite Stekloff operators. The main tool that we developed is a fast Fourier method for solving constant coefficient Helmholtz equation with Dirichlet or Neumann boundary condition on rectangular domain. The resulting algorithm is efficient, 


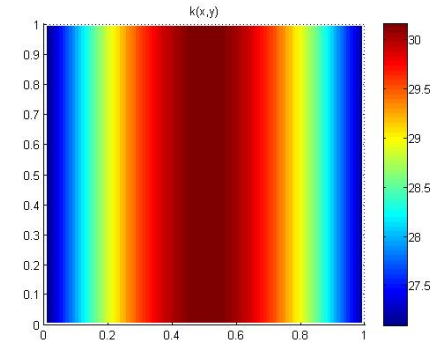

(a) wave number function $k_{1}(x, y)$

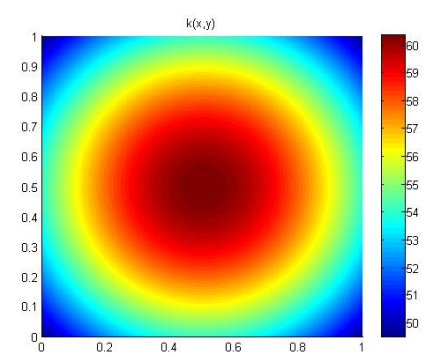

(c) wave number function $k_{2}(x, y)$

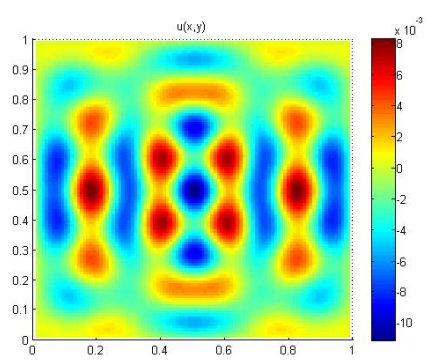

(b) wave simulated $u_{1}(x, y)$

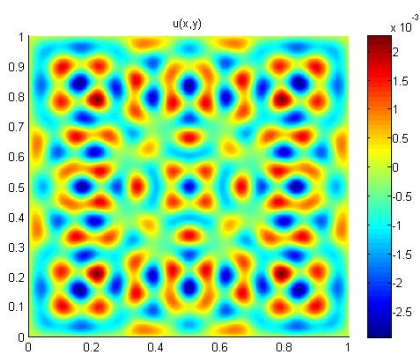

(d) wave simulated $u_{2}(x, y)$

Fig. 1. Simulation results for different velocity fields.

Table 2. Smallest six Stekloff eigenvalues in magnitude for different $\eta$

\begin{tabular}{|c|c|c|c|c|c|c|}
\hline$\eta$ & $\lambda_{1}$ & $\lambda_{2}$ & $\lambda_{3}$ & $\lambda_{4}$ & $\lambda_{5}$ & $\lambda_{6}$ \\
\hline 0.5 & 0.2132 & 0.2158 & 0.5561 & 0.8910 & 0.8910 & 2.6103 \\
\hline 1 & 0.2162 & 0.2190 & 0.5832 & 1.0003 & 1.0003 & 4.1828 \\
\hline 2 & 0.2302 & 0.2327 & 0.7548 & 2.4831 & 2.4831 & -2.6194 \\
\hline 4 & 0.4909 & 0.4909 & 0.6099 & 0.7888 & 1.5170 & 1.5170 \\
\hline
\end{tabular}

transparent, and easy to implement. Our numerical experiments show that such algorithm works also as a solver for the Helmholtz problem with mildly varying coefficient (non-constant wave number). Another pool of important applications will be the computation of transmission eigenvalues, where our method has the potential to provide an efficient computational tool. 


\section{Bibliography}

Achi Brandt and Irene Livshits. Wave-ray multigrid method for standing wave equations. Electron. Trans. Numer. Anal, 6(162-181):91, 1997.

F. Cakoni, D. Colton, S. Meng, and P. Monk. Stekloff eigenvalues in inverse scattering. SIAM J. Appl. Math., 76(4):1737-1763, 2016. ISSN 0036-1399. doi: 10.1137/16M1058704. URL http://dx.doi.org/10.1137/16M1058704.

Fioralba Cakoni and Rainer Kress. A boundary integral equation method for the transmission eigenvalue problem. Appl. Anal., 96(1):23-38, 2017. ISSN 0003-6811. doi: 10.1080/00036811.2016.1189537. URL http://dx.doi.org/ 10.1080/00036811.2016.1189537.

Zhiming Chen and Xueshuang Xiang. A source transfer domain decomposition method for Helmholtz equations in unbounded domain. SIAM Journal on Numerical Analysis, 51(4):2331-2356, 2013.

James W. Cooley and John W. Tukey. An algorithm for the machine calculation of complex Fourier series. Math. Comp., 19:297-301, 1965. ISSN 0025-5718.

Howard C Elman, Oliver G Ernst, and Dianne P O'leary. A multigrid method enhanced by krylov subspace iteration for discrete helmholtz equations. SIAM Journal on scientific computing, 23(4):1291-1315, 2001.

Björn Engquist and Lexing Ying. Sweeping preconditioner for the Helmholtz equation: moving perfectly matched layers. Multiscale Modeling $\mathscr{G}$ Simulation, 9(2):686-710, 2011.

Oliver G Ernst and Martin J Gander. Why it is difficult to solve Helmholtz problems with classical iterative methods. In Numerical analysis of multiscale problems, pages 325-363. Springer, 2012.

Mehran Eslaminia and Murthy N Guddati. A double-sweeping preconditioner for the Helmholtz equation. Journal of Computational Physics, 314:800-823, 2016.

M. J. Gander, I. G. Graham, and E. A. Spence. Applying GMRES to the Helmholtz equation with shifted Laplacian preconditioning: what is the largest shift for which wavenumber-independent convergence is guaranteed? Numer. Math., 131(3):567-614, 2015. ISSN 0029599X. doi: 10.1007/s00211-015-0700-2. URL http://dx.doi.org/10.1007/ s00211-015-0700-2.

Gene H Golub and Charles F Van Loan. Matrix computations, volume 3. JHU Press, 2012.

Daniel Osei-Kuffuor and Yousef Saad. Preconditioning helmholtz linear systems. Applied numerical mathematics, 60(4):420-431, 2010.

Christiaan C Stolk. A rapidly converging domain decomposition method for the Helmholtz equation. Journal of Computational Physics, 241:240-252, 2013.

Leonardo Zepeda-Núnez and Laurent Demanet. The method of polarized traces for the 2d Helmholtz equation. Journal of Computational Physics, 308:347$388,2016$. 